\title{
Non-adiabatic dynamic of atmospheric unimolecular photochemical reactions of 4,4-difluoro-crotonaldehyde using TD-DFT and TSH approaches
}

\author{
Pedro J Castro Pelaez ${ }^{1}$, Satoshi Maeda ${ }^{2}$, and Keiji Morokuma ${ }^{3}$ \\ ${ }^{1}$ Universidad del Atlantico \\ ${ }^{2}$ Hokkaido University \\ ${ }^{3}$ Kyoto University
}

February 9, 2021

\begin{abstract}
Photochemical reactions of small molecules occur upon irradiation by ultraviolet or visible light, and they are a very important and controversial chemical process in the Earth's atmosphere because they impact our quality of life and health. Smallunsaturated carbonyl compounds play an important role in the chemistry of the polluted troposphere. The fluorinated aldehydes are very reactive under the sunlight driving to species that trigger more atmospheric reactions. This paper is focused on a theoretical study of the photochemistry of difluoro-crotonaldehyde using static and dynamic calculations by combination of Global Reaction Route mapping (GRRM) and Trajectory Surface Hopping (TSH) approach. The static analysis of the electronic and geometrical structures at the critical points allowed to rationalize the possible pathways that interconnect the stationary and crossing points in order to get a map of the unimolecular photochemical reactions which take place. The time evolution of the electronic states and the degrees of freedom enabled the identification of the requirements to follow the most probable deactivation pathways. This article reports the unimolecular deactivation pathways after the electronic excitation of the trans and cis isomers. In both cases, the excitation energies were calculated and compared with the analogous in the crotonaldehyde in order to elucidate the effect of fluorine atoms on the electronic structure and stabilities. After the initial excitations to the $\pi \pi^{*}$ excited states, the main deactivation channels follow non-adiabatic pathways via $\mathrm{S}_{1} / \mathrm{S}_{0}$ conical intersections. Ultrafast processes leading to the early activation of the $\mathrm{S}_{1}$ govern the decay of the difluoro-crotonaldehyde. Depending on the nature of the $\mathrm{S}_{1}$ state before the crossing with the $\mathrm{S}_{0}$, the system can follow several reaction pathways. The main photochemical processes observed were the cis-trans isomerization, the Norrish type I reaction ( $\alpha$-cleavage), Norrish type II reaction ( $\gamma$-hydrogen abstraction) and fluorine photodissociation. The time scale, the molecular deformations and the electronic states implied for the different photochemical processes, as well as how these compete with the photophysical deactivation are discussed.
\end{abstract}

\section{Hosted file}

PCastro_Manuscript.pdf available at https://authorea.com/users/394520/articles/508083-nonadiabatic-dynamic-of-atmospheric-unimolecular-photochemical-reactions-of-4-4-difluorocrotonaldehyde-using-td-dft-and-tsh-approaches

\section{Hosted file}

PCastro_Manuscript_Images.pdf available at https://authorea.com/users/394520/articles/508083non-adiabatic-dynamic-of-atmospheric-unimolecular-photochemical-reactions-of-4-4difluoro-crotonaldehyde-using-td-dft-and-tsh-approaches

\section{Hosted file}


PCastro_Manuscript_Tables.pdf available at https://authorea.com/users/394520/articles/508083non-adiabatic-dynamic-of-atmospheric-unimolecular-photochemical-reactions-of-4-4difluoro-crotonaldehyde-using-td-dft-and-tsh-approaches 